\title{
The Design of a Heavy-load Palletizing Robotic Structure for Coating Handling \\ Weixiong $\mathrm{Xu}^{1, \mathrm{a}}$, Jing $\mathrm{CaO}^{1, \mathrm{~b}}$, Xiaogeng $\mathrm{Ni}^{2, \mathrm{c}}$ \\ ${ }^{1}$ Palletizing Robots Engineering Center of universities in fujian province, China \\ ${ }^{2}$ Fuzhou University, China \\ 12308798018@qq.com, b1606862556@qq.com, cnxg@fzu.edu.cn
}

Keywords: Palletizing, Robot.

\begin{abstract}
Heavy load palletizing robot in solving labor shortage, improve labor productivity, reduce production cost and the labor intensity of workers, improve the production environment, etc. It is of great significance. This article expounds the heavy load of palletizing robot working principle, structure and main structure design; using the finite element analysis software Solidworks Simulation for robot arm structure of dynamic and static mechanics analysis; Palletizing robot motion analysis. A large range of prototype experiments show that the robot movement, carrying capacity is strong, fast and can meet the design requirements.
\end{abstract}

\section{Introduction}

Stacking technique is a new technology of modern logistics automation, palletizing robot, turned a piece of a product or material stacking according to certain rules into the crib, easy to handle storage, to lift the heavy labor, improve labor productivity and improve the production environment is of great significance., along with the development of science and technology progress and palletizing robot application for palletizing robot not only has rapid accurate handling material, also calls for palletizing robot with overload, high efficiency, high reliability and low cost etc. The three trees paint company is one of the enterprises, the local maximum paint bucket weight $200 \mathrm{~kg}$, the production line has been using artificial handling the bucket, the labor intensity of workers, environments, and there is a big security hidden danger. The three trees paint company designed and developed a kind of used for coatings, carrying the heavy load of palletizing robot, implements the enterprise production process of the bucket handling automation. In this paper, the structure composition and the main structure design.

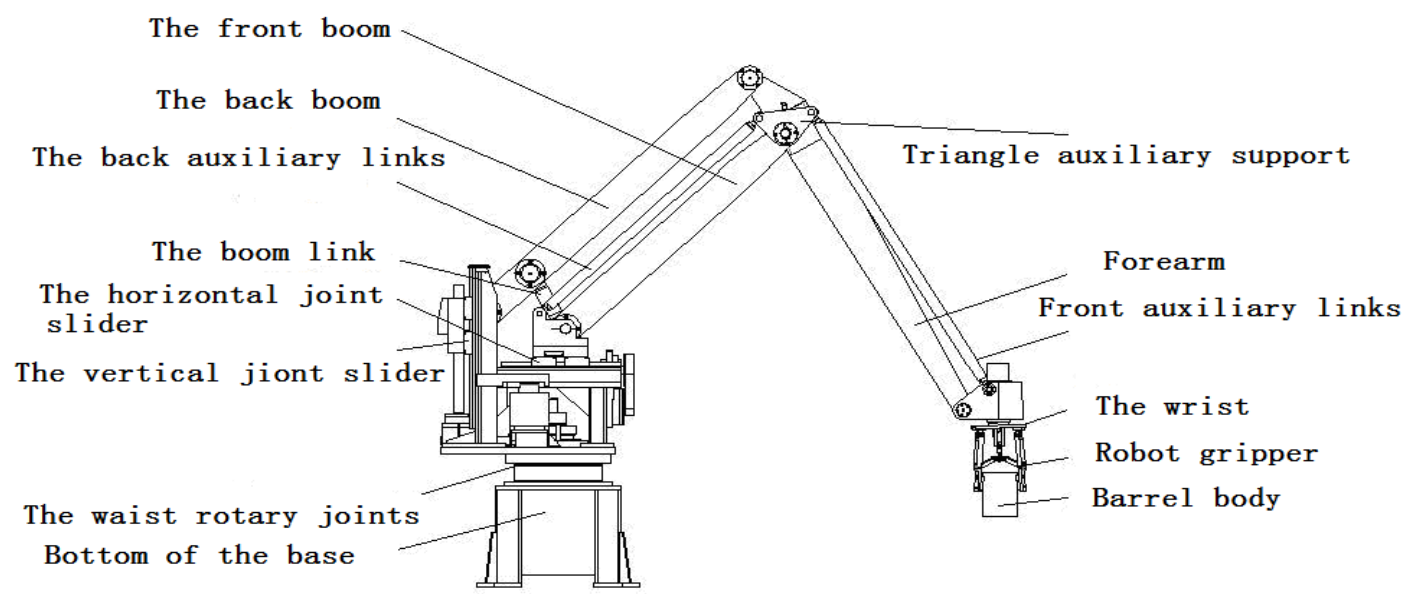

Fig. 1 The overall structure of palletizing robot. 


\section{The Overall Structure of Palletizing Robot}

Palletizing robot is four degrees of freedom robot, whose entire equipment consists of mechanical system, driving part and control sensing device. The mechanical system is divided into four parts, namely bottom of the base, waist, arm and wrist. The bottom of the base is fixed on the ground as installed base. Waist can drive the rotary motion of the robot. Arm mainly includes forearm, the front boom, the back boom and auxiliary linkage. The front boom bottom of horizontal motion and the back boom bottom of up-and-down motion can drive the end of the forearm to move front and back, up and down. Wrist, the installed position of manipulator, controls the rotational positioning of the manipulator by the motor [1]. The structure details are shown as Fig. 1.

\section{The Structure Design of the Arm}

Arm is used to implement the wrist in different position of plane motion. the front boom, the back boom and auxiliary linkage form a parallelogram mechanism between forearm. Horizontal servo motor through the synchronous belt drives the ball screw, screw drive the front boom level slider along the horizontal direction. By the same token, the vertical guide direction of the servo motor through the synchronous belt drives the vertical ball screw, vertical screw drive the back boom horizontal slider along the vertical guide direction.

Forearm at the end of the plane coordinates of $\mathrm{x} \mathrm{z}$, is depends on horizontal direction and vertical direction of the movement of the servo motor position. Palletizing robot orientation in the $\mathrm{x}, \mathrm{z}$ plane, is the change of the shape of a parallelogram witch composed of the front boom, the back boom and auxiliary linkage and forearm.

It is worth mentioning the forearm auxiliary connecting rod, forearm, triangle auxiliary support, wrist and form a parallelogram, the front boom arm and connecting rod, the triangle auxiliary support, horizontal slider is another line of quadrilateral formation, to ensure that the two parallelogram wrist in the process of up and down or horizontal movement in the level and did not tilt.

The structure details are shown as Fig. 1 and Fig. 2.

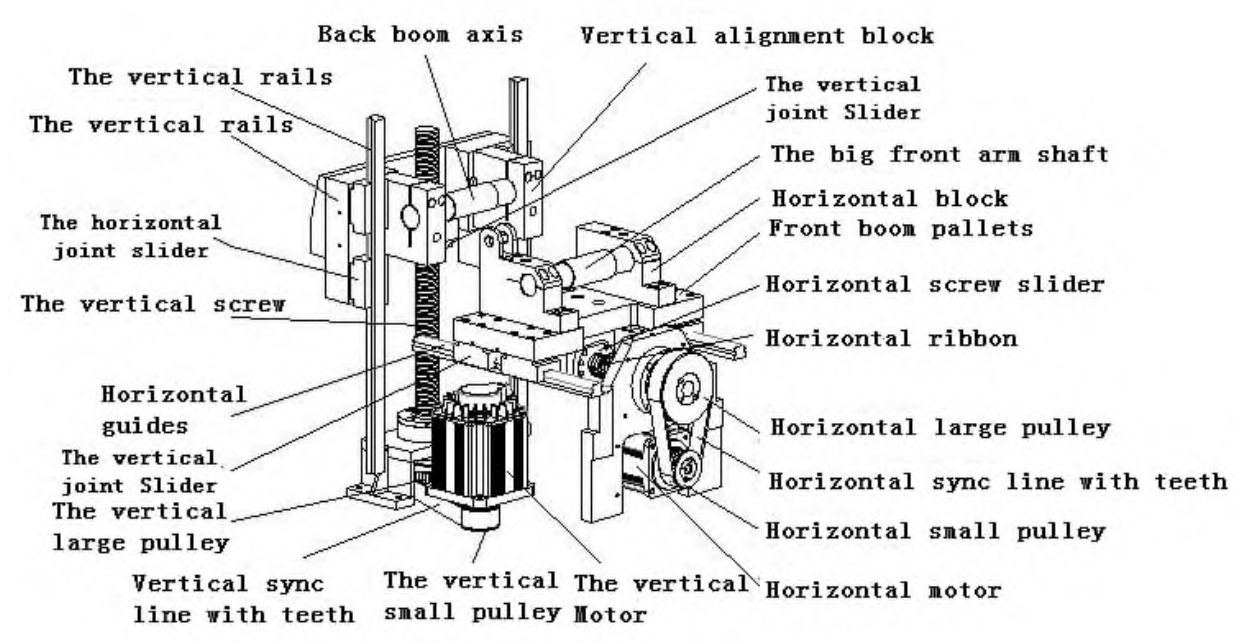

Fig. 2 The structure design of the arm.

\section{The Structure Design of the Waist}

The waist is arm bearing parts, it's for rotary parts, rotary movement of the waist and plane movement of the arm, realize the wrist grabs the accurate positioning of the space, the waist of the rotary motion needed motor through the synchronous cog belt drive and fixed on the base at the bottom of the gear, using big reduction ratio of gear deceleration increasing twist can overcome the 
arm, and a large floor the moment of inertia of the driving part. The structure design of the waist is as shown in Fig. 3.

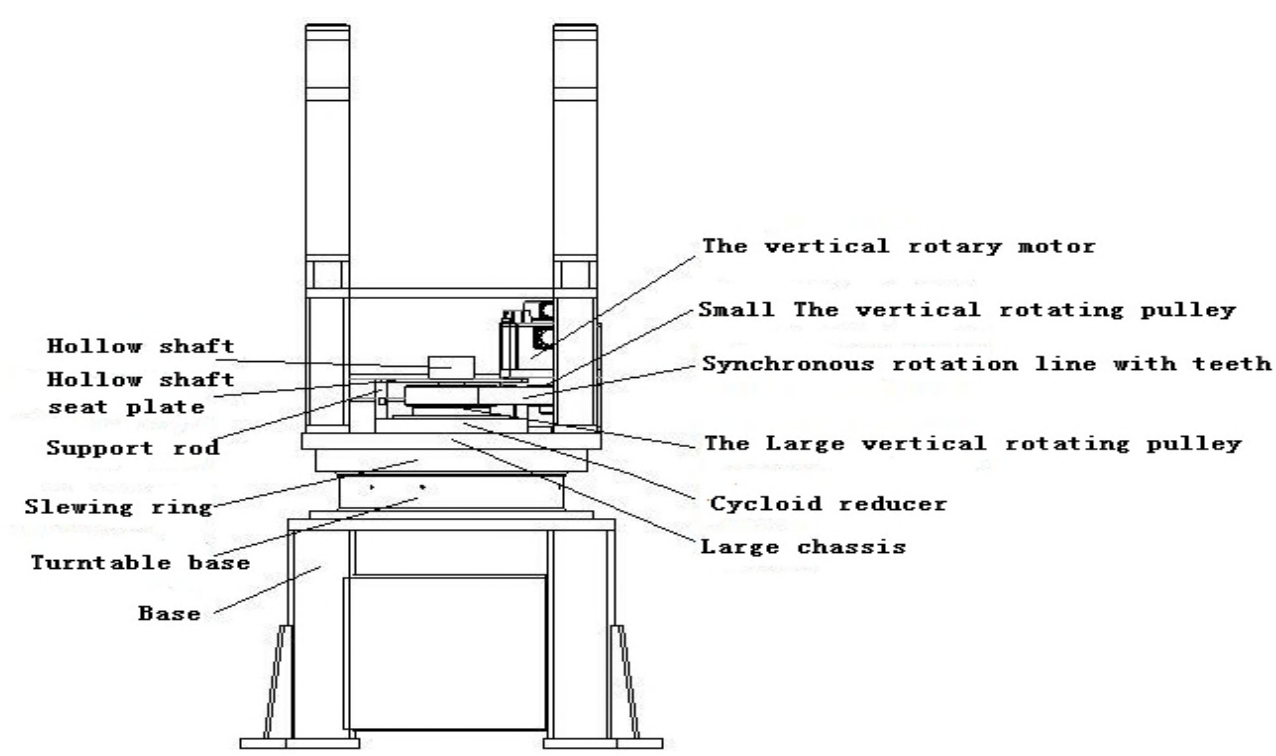

Fig. 3 The structure design of the waist.

Cycloidal pin gear speed reducer, a new arranger adopted planetary transmission principle and cycloidal pin gear speed reducer tooth mesh, featuring high transmission efficiency and high transmission ratio, is mainly used in heavy load robot and engineering machinery.

Suppose the input rotate speed of high speed shaft of cycloidal pin gear speed reducer as $n$, low speed shaft fixed on the bottom dock and the chassis as output to drive big raft swing of the wrist, the reduction ratio can be expressed as follows:

$$
i=\frac{1}{n+1}
$$

The swing movement of big raft depends on large-size bearing, which is a large bearing that can endure combined load.

The bearing, composed of mounting holes, internal gear or gear, grease holes and sealing device, can ensure the compact size of main engine, easy guide and maintenance

Reduction gear adopts FC-A65 cycloidal reducer, while slewing bearing applies Type 010.25.400 of JB/T2300-1999 Standard. The structure details are shown as Fig. 3.

\section{The Structure Design of the Wrist}

Positioning hole on the wrist can install all kinds of grabs, so that the pallet all kinds of material, can install vacuum suction cup, can install packaged materials, etc.

Equipped with servo motor and reducer on the wrist, waist and arms to catch with the spatial orientation, and the wrist is used to determine the direction of fetching or release material.

In this paper, case for the barrel material of grabs. The scratching in the operation of a main complete pallet put barrel motion of the object, grabs by four claws, connecting rod, universal joint, and the tensile cylinder, when the cylinder contraction, by universal joint taut four connecting rod, a robot grabs four claw is fasten barrel material, as shown in Fig. 4, in order not to hurt the fetching material, equipped with rubber on robot grabs claw, can fasten the material not only so, don't hurt $f$ 


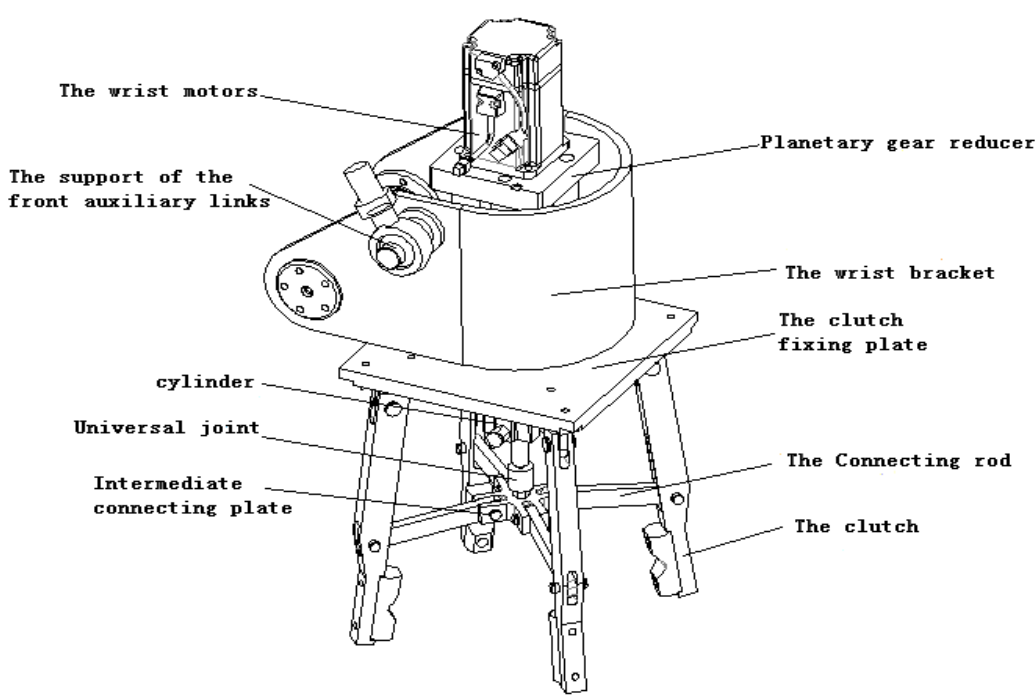

Fig. 4. The structure design of wrist.

\section{The Wrist Motor and Reducer Type Selection Calculation}

The function of the wrist is by adjusting the Angle of the servo motor, determine the scraping or place the direction of the material [2]. Wrist load consists of two parts, grabs and fetching material, when the wrist motor to overcome the load torque, torque including generated by the friction torque and load produced by the rotation inertia moment, and the drag torque is generated by the friction of bearing rolling friction, its value is very small and negligible, wrist rotary inertia of the load on the main consideration grab materials. The bucket is one of the biggest diameter of $800 \mathrm{~mm}$, maximum height of $1000 \mathrm{~mm}$ cylinder, the moment of inertia of its relative to the motor rotation is:

$$
J=\frac{1}{2} m R^{2}
$$

$\mathrm{R}$ refers to the diameter of the barrel, $\mathrm{R}=800 \mathrm{~mm}$

$\mathrm{M}$ refers to the weight of packing bag, $\mathrm{m}=100 \mathrm{~kg}$

The calculation can be $\mathrm{J}_{1}=8 \mathrm{~kg} \cdot \mathrm{m}^{2}$. Grabs around the rotational inertia of the motor shaft by Solidworks calculation for: $J_{2}=1.2653 \mathrm{~kg} / \mathrm{m}^{2}$, the wrist is the total moment of inertia of the load: $\mathbf{J}$ $=\mathrm{J}_{1}+\mathrm{J}_{2}=8+1.2653=9.2653 \mathrm{~kg} \cdot \mathrm{m}^{2}$

By the inertia moment

$\mathrm{T}=\mathrm{J} \times \varepsilon$

Type: $\varepsilon$ - grabs the rotation of the acceleration,

Grasp the maximum acceleration of rotation: $\varepsilon=4 \pi \mathrm{rad} / \mathrm{s}^{2}$

Then $\mathrm{T}=\mathrm{J} \times \varepsilon=9.2653 \times 4 \pi=116.37 \mathrm{~N} \cdot \mathrm{m}$

Wrist selected and used Zhongda diantong co., Production of servo motor, type of ECMA C10807RS, output torque: $2.39 \mathrm{~N} \cdot \mathrm{m}$, output speed : $3000 \mathrm{r} / \mathrm{min}$. Choose hubei planetary transmission equipment co., the production of planetary gear reducer, model PH120 - LB, ratio of 100:1.Palletizing robot run shows that can satisfy grabs requirements.

\section{Finite Element Analysis based on Solidworks Simulation}

Simulation of finite element analysis software is a seamless plug-in of Solidworks software, can provide static analysis, heat transfer, distortion, frequency analysis [3]. Simulation is used to analyze the arm assembly body static analysis, it is concluded that the arm member strength and stiffness analysis of the results. First set up the mathematical model of connecting rod, forearm, the front boom, the back boom and auxiliary linkage, set the parts material, sets the parts connected to 
pin connector, to the highest point for the stress analysis of dangerous fixed the front boom, the back boom at the bottom of the hole, choose a suitable unit type and size of mesh, Perform software and analysis stress distribution and displacement distribution are obtained. Can find the forearm with the front boom connecting hole to maximize stress.

Through the analysis of the finite element simulation, can satisfy the requirements of the use of member strength and stiffness, rationalize the structural dimension, in order to reduce weight, reduce the moment of inertia, save material, reduce the manufacturing cost.

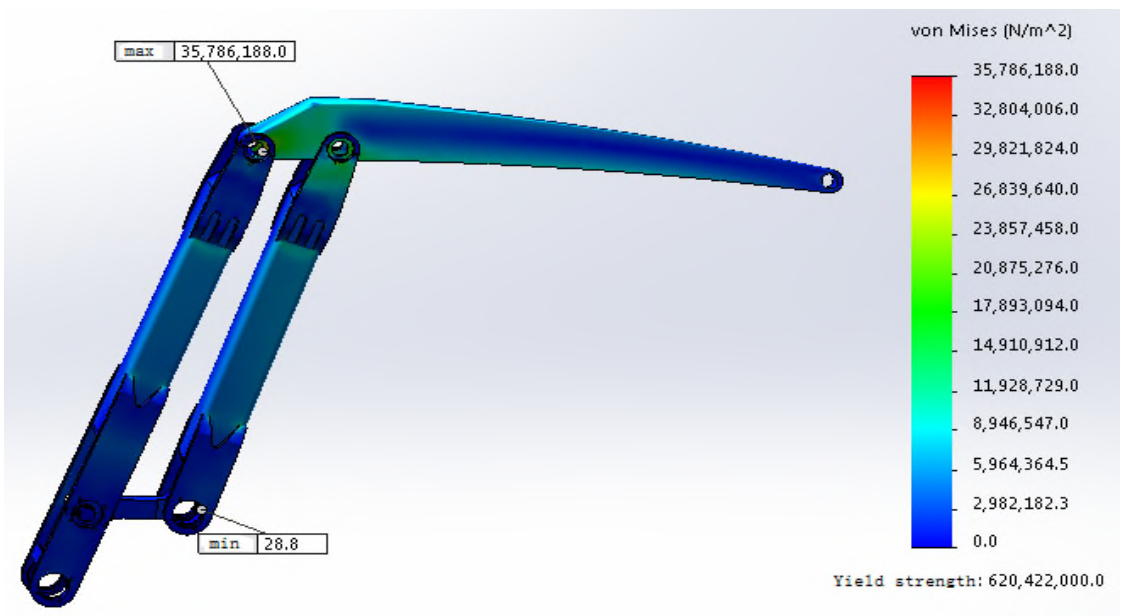

Fig. 5 The stress distribution of arm components.

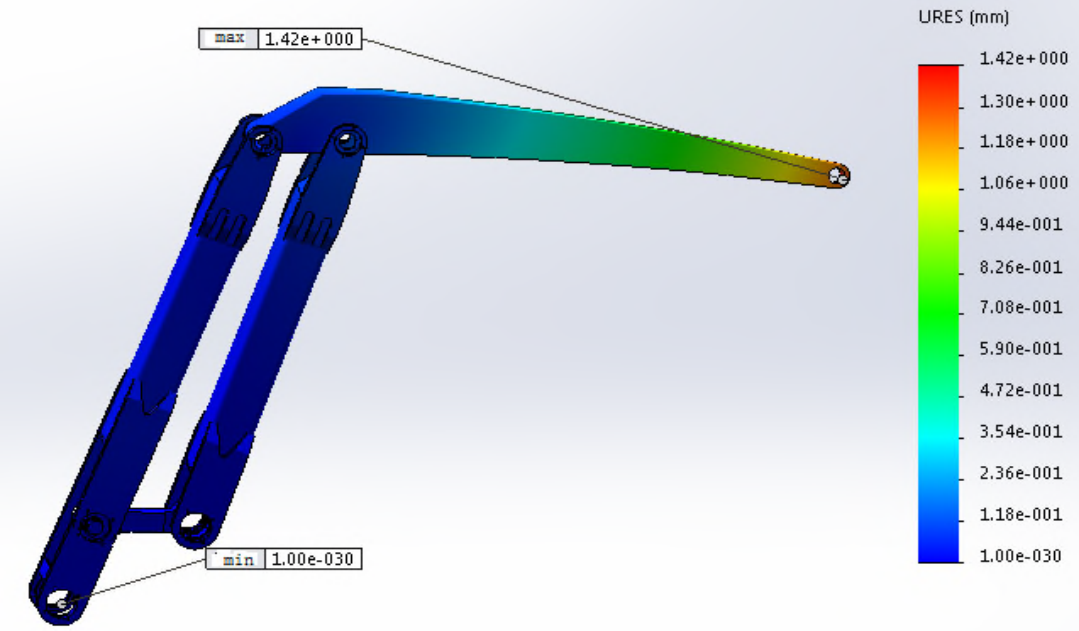

Fig. 6 The displacement distribution of arm components.

\section{Motion Analysis on Palletizing Robot}

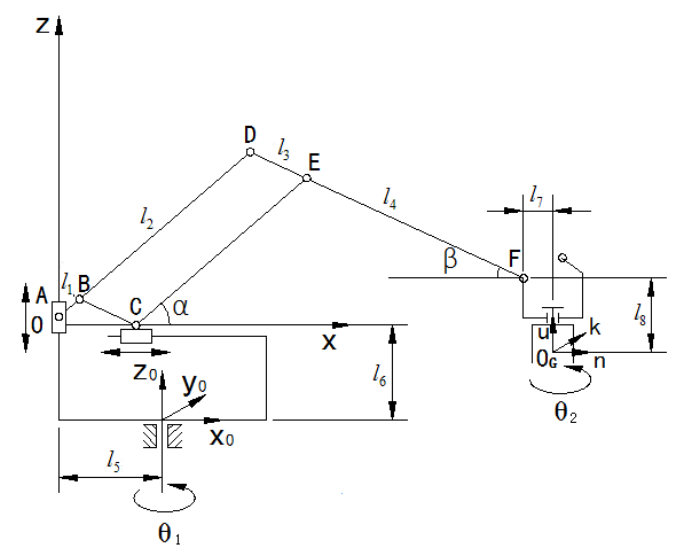

Fig. 7 Kinematics analysis on palletizing robot. 
Palletizing robot kinematics analysis to establish a coordinate system based on the analytic geometry method, analyze its kinematics characteristic, calculate the actuator at the end of the position and posture with various depending on a parameter, the relationship between map diagrams f palletizing robot kinematics [4].

Among : $1_{1}=280,1_{2}=1400,1_{3}=320,1_{4}=1600$ 。

The position of A point coordinates:

$\mathrm{A}:\left[\begin{array}{l}x_{A} \\ z_{A}\end{array}\right]=\left[\begin{array}{c}l_{3} \sin \beta-l_{1} \sin \alpha \\ 0\end{array}\right]$

The position of B point coordinates:

$\mathrm{B}:\left[\begin{array}{l}x_{B} \\ z_{B}\end{array}\right]=\left[\begin{array}{c}l_{1} \cos \alpha \\ z_{A}+l_{1} \sin \alpha\end{array}\right]$

The position of $\mathrm{C}$ point coordinates:

$\mathrm{C}:\left[\begin{array}{l}x_{C} \\ z_{C}\end{array}\right]=\left[\begin{array}{c}l_{1} \cos \alpha+l_{3} \cos \beta \\ 0\end{array}\right]$

The position of $\mathrm{E}$ point coordinates:

$\mathrm{E}:\left[\begin{array}{c}x_{E} \\ z_{E}\end{array}\right]=\left[\begin{array}{c}x_{C}+l_{2} \cos \alpha \\ l_{2} \sin \alpha\end{array}\right]$

The position of $\mathrm{F}$ point coordinates:

$\mathrm{F}:\left[\begin{array}{l}x_{F} \\ z_{F}\end{array}\right]=\left[\begin{array}{l}x_{E}+l_{4} \cos \beta \\ z_{E}-l_{4} \sin \beta\end{array}\right]$

Joint (3) to (8) obtain:

$\left[\begin{array}{c}x_{F} \\ z_{F}\end{array}\right]=\left[\begin{array}{c}\left(1+\frac{l_{2}}{l_{1}}\right) x_{c}+\left(l_{4}-\frac{l_{2} l_{3}}{l_{1}}\right) \cos \beta \\ \left(l_{2}-\frac{l_{4} l_{1}}{l_{3}}\right) \cos \alpha-\frac{l_{4}}{l_{3}} z_{A}\end{array}\right]$

In order to solve the convenience, make: $l_{1} l_{4}=l_{2} l_{3}$

$\left[\begin{array}{l}x_{F} \\ z_{F}\end{array}\right]=\left[\begin{array}{c}\left(1+\frac{l_{2}}{l_{1}}\right) x_{c} \\ -\frac{l_{4}}{l_{3}} z_{A}\end{array}\right]$

The position of $\mathrm{G}$ point coordinates:

$\left[\begin{array}{c}x_{G} \\ z_{G}\end{array}\right]=\left[\begin{array}{c}\left(1+\frac{l_{2}}{l_{1}}\right) x_{c}+l_{7} \\ -\frac{l_{4}}{l_{3}} z_{A}-l_{8}\end{array}\right]$

$\left[\begin{array}{c}x_{O G} \\ y_{O G} \\ z_{O G} \\ 1\end{array}\right]=\left[\begin{array}{c}{\left[\left(1+\frac{l_{2}}{l_{1}}\right) x_{C}+l_{7}-l_{5}\right] \cos \left(\theta_{1}+\theta_{2}\right)} \\ {\left[\begin{array}{c}l_{2} \\ l_{1}\end{array} x_{C}+l_{7}-l_{5}\right] \sin \left(\theta_{1}+\theta_{2}\right)} \\ -\frac{l_{4}}{l_{3}} z_{A}-l_{6}-l_{8} \\ 1\end{array}\right]$ 
Formula 12 is the equation of motion for palletizing robot at $\mathrm{G}$ point. Palletizing robot can realizes precise positioning by controlling the horizontal motion mechanism and vertical motion mechanism of servo motor and changing the value of $\alpha, \beta$ and $\theta_{1}, \theta_{2}$. Driven by servo motor horizontal motion mechanism and vertical motion mechanism [5].

\section{Conclusion}

This article from the working principle, structure composition, main structure design, finite element analysis software Solidworks and used for coating are introduced based on the analysis of the movement to carry the heavy load of palletizing robot. Prototype test results show that the robot is of compact structure, flexible motion, large carrying capacity, good manufacturability, manufacturing and assembly of convenient operation, accurate quickly completed the multi-layer stacking barrel coating test, better meet the needs of the finished product in production line handling.

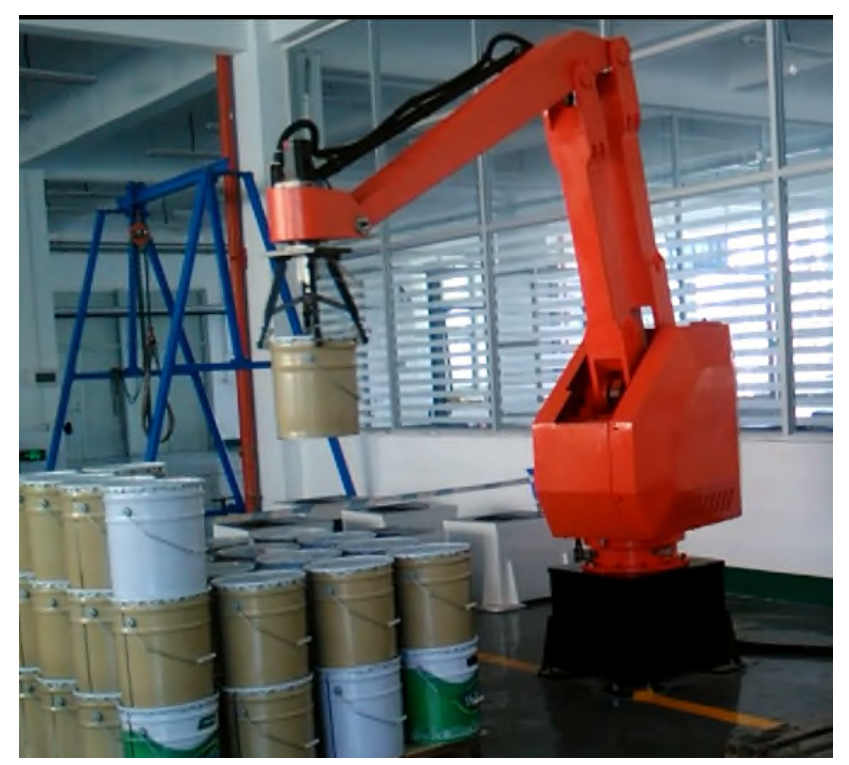

Fig. 8 The situation of the palletizing robot operating.

\section{References}

[1] L. A. Zhang, X. L. Yu, J. S. Zhao, School of Mechanical Engineering Anhui University of Technology Maanshan; ChinaJiang-ping MEI School of Mechanical Engineering Tianjin University Tianjin; China, Analysis, Contol Software Design and Workshop Application of a Robot Palletizing Line; 2010 2nd International Conference on Intellectual Technology in Industrial Practice (ITIP2010).

[2] H. X. Yu, School of mechanical engineering Ningxia University Yinchuan; China Junfeng Shan; and Xuejun Zhu School of mechanical engineering Ningxia University Yinchuan; China, Off-line programming and remote control for a palletizing robot, 2011 IEEE International Conference on Computer Science and Automation Engineering (CSAE 2011).

[3] V. Graefe, R. Bischoff, From Ancient Machines to Intelligent Robots A Technical Evolution From Ancient Machines to Intelligent Robots A Technical Evolution. 2014 IEEE International Conference on Progress in Informatics and Computing.

[4] Japan Robot Association, Robotics Manual, Science Press, Beijing, 1996.

[5] Y. Wang, J. W. Luo, W. B. Li, H. Z. Tan; Y. N. Zhang, Inverse-Free D1G1 Solution toAcceleration-Level Inverse Kinematics of Redundant Robot Manipulators, 2014 IEEE International Conference on Progress in Informatics and Computing. 